\title{
Etiology of Adolescent Idiopathic Scoliosis: A Literature Review
}

\author{
Sina Rashidi Kikanloo, Sandip Parshottam Tarpada, Woojin Cho \\ Department of Orthopedic Surgery, Albert Einstein College of Medicine/Montefiore Medical Center, Bronx, NY, USA
}

\begin{abstract}
Adolescent idiopathic scoliosis (AIS) is the peripubertal development of spinal curvature of a minimum of $10^{\circ}$. AIS is thought to be attributable to genetic factors, nutrition, early exposure to toxins, and hormonal dysregulation. Recent literature suggests these factors may compound to determine both disease onset and severity. Currently, treatment is limited to observation, bracing, and surgical intervention. Intervention is presently determined by severity and risk of curve progression. As they emerge, new therapies may target specific etiologies of AIS.
\end{abstract}

Keywords: Adolescent; Scoliosis; Adolescent idiopathic scoliosis; Etiology; Cobb angle; Review

\section{Introduction}

Adolescent idiopathic scoliosis (AIS) is the most common type of scoliosis affecting $2 \%-4 \%$ of adolescents, mainly involving children between 10 and 18 years of age [1]. AIS has both congenital and developmental components, with the former being studied from the perspective of genetic variation. Using comparative genetic methodologies, such as genome-wide association studies (GWAS), several genomic linkages have been shown to be associated with this spine pathology. Thus, it can be determined that AIS is polygenic or a quantitative trait locus, which can vary due to several different genetic loci. The etiology is not limited to genetic predispositions but has been studied in association with physiological, anatomical, and hormonal disruptions. The literature has been reviewed to provide a holistic understanding of AIS etiology.

\section{Genetics}

Genetic predisposition has been one of the most studied aspects of AIS development, and research on this topic has focused on several loci in the growth physiology. Andersen et al. [2] analyzed Vang-like protein 1 (VANGL1) gene mutations in the coding region in 157 patients with AIS and compared them with control subjects. Two major mutations, p.I136N and p.F440V, disrupting Wingless/Int1 ligands (WNT)/Planar Cell Polarity signaling were observed [2]. It is hypothesized that these mutations disrupt the translocation of VANGL1 receptors to the membrane, but the exact pathophysiological mechanism was not determined in the study. These mutations, although rare in the general population, present with high levels of penetrance in this patient group. Therefore, it is believed that VANGL1 mutations can predispose individuals to AIS in later life.

Received Apr 22, 2018; Revised Jul 23, 2018; Accepted Aug 15, 2018

Corresponding author: Woojin Cho

Department of Orthopedic Surgery, Multidisciplinary Spine Group, Albert Einstein College of Medicine/Montefiore Medical Center, 3400 Bainbridge Ave, 6th Floor, Bronx, NY 10467, USA

Tel: +1-718-920-5376, Fax: +1-718-654-2396, E-mail: wcho@montefiore.org 
In a similar pathway to VANGL1, the calmodulin 1 (CALM1) gene has been associated with the development of AIS. Three single nucleotide polymorphisms (SNPs) in the CALM1 gene, rs2300496, rs2300500, and rs3231718, were associated with AIS development, but these did not affect the main curve, severity, or genotypic distribution [3]. The CALM1 protein plays a crucial role in ion pores and platelets, and CALM1 gene mutations have been linked to long QT syndrome and ventricular tachycardia. However, SNPs at these sites, while not necessarily pathological in cardiac development, can play a role in musculoskeletal development $[4,5]$. Genetic disruption in platelet development is not limited to CALM1. Fibrillin-1 and fibrillin-2 mutations have also resulted in severe spinal curvature [6]. Therefore, it is clear that AIS comorbidity with other diseases, such as long QT syndrome and ventricular tachycardia, is useful as a clinical tool to understand the cardiac issues that may arise because of a spinal abnormality.

Collagen and the extracellular matrix play a role in the development of AIS through impaired growth. A growth imbalance, rather than anatomical differences, is the imperative distinction in the pathophysiology of curve development and progression [7]. These imbalances, in some cases, are genetic. Haller et al. [8] observed a missense mutation at the collagen type XI alpha 2 chain (COL11A2) in $32 \%$ of patients with AIS. Although the mutation does not present in most cases, mutations of the extracellular matrix that become exacerbated through rapid growth may result in a pathophysiological disruption of spinal alignment.

Specific populations have been studied to identify genetic predispositions for idiopathic scoliosis. In the study by Grauers et al. [9], exome sequencing and analysis for novel or rare missense, nonsense, or splice site variants were performed for 1,739 patients and 1,812 control subjects. The sequence of ladybird homeobox 1 ( $L B X 1)$, although not initially analyzed by exome sequence, was Sanger sequenced, and a variant was observed downstream of the LBX1 gene. The intergenic variant, rs11190870, showed a highly significant association to idiopathic scoliosis. In addition, the study did not find variants in the 5' UTR, noncoding exon, and promotor regions that showed a significant link to the pathology [9]. Limited to the Scandinavian population and including variations of idiopathic scoliosis, the study could not directly provide an etiology linked to AIS. However, it provided more evidence for ge- netic etiology for the variations in the general pathology. Moreover, the study linked specific genetic variations of the pathology to different populations, thereby providing evidence for the polygenic etiology of AIS.

In the study by Zhu et al. [10], LBX1 was further suspected for its involvement in the asymmetrical expansion of the WNT/beta catenin pathway. Beta catenin, $T N I K$, and $L B X 1$ mutations were linked to disruptions in the paraspinal muscle development regulated by the WNT/beta catenin pathways. Guo et al. [11] further observed LBX1b gain of function mutations downregulated WNT5b, resulting in the displaced dorsal melanophore stripe and notochord deformation in zebrafish models. This further links the aforementioned genetic dysregulation of imperative developmental pathways regarding adolescent growth. Pathophysiological development will be discussed in more detail in a later section of this review.

To elaborate on the genetic variations in other populations, GPR126 polymorphism has been associated with AIS in Chinese populations. Although the literature is limited in its analysis of the gene in other populations, SNPs within GPR126, specifically rs6570507 A $>\mathrm{G}(p=0.0035$; odds ratio [OR], 1.729), rs7774095 $\mathrm{A}>\mathrm{C}(p=0.0078$; OR, 1.687), and rs7755109 $A>G(p=0.0078$; OR, 1.687), have been linked with AIS development. Specifically associated with the adolescent variant of idiopathic scoliosis, researchers believe that the affected G-protein receptor 126 plays a role in musculoskeletal development, and thus, its disruption plays a role in the AIS pathogenesis [12].

In a Chinese family, a quantitative polymerase chain reaction study identified the mutation c.2645A $>C$ (p.E882A) within the AKAP2 gene as associated with AIS phenotypes. Located on linkage locus IS4 on chromosome 1, the gene had been previously implicated in skeletal development. The study showed that in pathological individuals, the mRNA expression was significantly increased compared to individuals not expressing the AIS phenotype [13]. Although limited to one family, the analysis of this gene has increased suspicion of the gene, and it is believed that further studies should be conducted to validate their results.

In a GWAS study of the Chinese Han population, several more genes were associated with susceptibility to AIS. Four SNPs in the BIN1, CDH13, SETBP1, and SPATA21 genes were associated with the adolescent development of scoliosis in this population [14]. On a larger scale, this study provides further evidence that AIS development is 
a multifactorial disease involving disruptions in either or both musculoskeletal and neurophysiological pathways.

In a study analyzing the role of transforming growth factor beta-1 (TGFB1) in a Russian population, it was determined that two common genetic polymorphisms, rs1800469 and rs1800471, increased the risk of AIS development in both male and female patients. Furthermore, the study concluded that rs 1800469 played a sexually dimorphic role in female and male subjects. The mutation was linked to earlier onset and severity of curvature in female patients, but not in male patients [15]. Thus, particular genes appear to lead to developmental abnormalities that manifest uniquely among male and female populations. TGFB1 was replicated in a Chinese population, and the gene was not associated with AIS predisposition in this population $[16,17]$. However, in patients with AIS, the severity of the curve was increased in those who carried the aforementioned mutations. One can see that the same genetic variation can play different roles in two different populations. Therefore, genetic interactions play a role in the multifaceted development of this disease.

With regard to treatments for AIS, the specific curvature type encountered influences treatment success. Genetic predispositions may determine bracing efficacy by reducing the prevalence of surgical intervention. A commercial GWAS genetic program testing 53 SNPs has been used to determine bracing efficacy and at-risk patients before the development of spinal abnormalities. These SNPs made it 16 times more likely to predict low-risk patients, and 5 times more likely to predict high-risk patients $[18,19]$. These SNPs were also able to determine the efficacy of bracing for patients; thus, predisposed individuals are more responsive to bracing treatment [20]. In evaluations of these SNPs, studies analyzed French-Canadian, Japanese, and Chinese Han populations for curve progression and bracing efficacy, and patients expressing these SNPs did not display greater bracing efficacy or differences in curve progression [14,21,22].

Genetic predisposition can play a crucial role in neurophysiology and musculoskeletal development. Although different populations have presented different genetic predispositions, genes play an important role in the development of the disease. These population differences further the notion that AIS is multifactorial in its development, and variances in other developmental genes may protect a population from AIS progression. Additionally, diet, toxins, and other nurturing elements have been associated with the development of AIS.

\section{Environmental Factors}

\section{Estrogen and melatonin}

Genetics, while a crucial facet of AIS development, cannot be the sole consideration in the development of AIS. In the study by Simony et al. [23], Cobb angles greater than $10^{\circ}$ were observed in twins using the Danish Twin Registry. A concordance rate of $0.40(0.10-0.70)$ was observed in monozygotic twins and a rate of 0.05 ( -0.05 to 0.15$)$ in dizygotic twins [23]. Thus, genetics, while imperative for the determination of AIS, does not entirely contribute toward the pathological development of the disease.

Estrogen and melatonin have long been controversial in the development of AIS and the severity of spinal curvature. In the study by Kulis et al. [24], concentrations of follicle-stimulating hormone, luteinizing hormone, estradiol, and progesterone were significantly lower in postmenarcheal female adolescents than in their control counterparts. However, in the study by Zhou et al. [25], higher serum estrogen levels and abnormal post-binding cellular responses were observed in patients with AIS. According to more recent studies, estrogen receptor- 2 polymorphism and asymmetrical expression that were previously believed to be associated with abnormal spine curvature are not associated with AIS development or severity.

While serum melatonin levels are not significantly different with initial diagnosis in the AIS population, melatonin levels decrease significantly with increased curvature progression [26]. Furthermore, melatonin receptor 1A/1B polymorphisms do not seem to be associated with AIS prevalence or severity, but certain polymorphisms in these receptors have been associated with the development of AIS. The role of receptors continues to remain controversial [27-30]. Thus, it is believed that serum melatonin levels, and possibly receptors, play a crucial role in the development of AIS. In an animal model, it was further confirmed that pinealectomy-induced melatonindeficient rats had issues with mechanical spinal loading and rotational instability like patients with AIS, further supporting the role of melatonin in AIS development [31]. Pinealectomies in chicken models manifested increased spinal deformities during development after hatching. Pinealectomies earlier in development rather than later resulted in much higher levels of spinal abnormalities, 
thus playing a role in early physiological development [32]. This procedure, however, did not result in spinal abnormalities in rodent and primate models, which was believed to be a result of inadequate stress on the spine due to lack of movement by these animals [32].

Estrogen and melatonin, two crucial hormones in growth regulation and adaptation, are controversial targets for therapy in adolescent populations. Further research is needed to fully understand the pathophysiological progression of the signal disruption in patients with AIS, and targeted patient-specific genetic and hormonal therapies can be used to address this subset of patients.

\section{Diet}

Environmental triggers of genetic predispositions must then be considered in the etiological processes of adolescent development of pathology. Gene-diet interactions have been observed in multiple facets of growth development; most profoundly, in adolescent populations during puberty. In Jamaica, these profound dietary influences were observed post-1965 with the addition of endocrine additives to livestock. After a 10-year delay, the incidence of scoliosis was dramatically increased. Until 1983, these rates increased until finally decreasing [33]. The Dutch Hunger Winter of 1944 had similar effects on the malleable developing population, and the epidemiological mechanisms are hypothesized to be similar. DNA methylation and epigenetic factors are considered the primary mechanism behind these changes, because these epigenetic factors can trigger genetic predispositions. Specifically, dietary components that are suspected to play a role include methyl donors, bioactive polyphenols, zinc, selenium, and vitamin A [34].

Alteration of the body composition can be seen in patients with AIS. In the study by Ramirez et al. [35], patients with AIS displayed lower body mass index (BMI) values, lower levels of fat composition, and lower fat-free mass indices. Hormonal dysregulation is believed to play a role in this, but there is a possible link between AIS and anorexia nervosa. Treatment to improve patient nutrition may delay exacerbation of spinal curvature in concordance with bracing and other common treatments.

\section{Exercise}

In a preliminary longitudinal case-controlled study, AIS was negatively associated with participation in dance, skating, gymnastics, karate, football, and hockey, suggesting preventative properties [33]. However, this study did not specify whether these activities were preventative measures or whether debilitation of the pathophysiology deterred involvement in these activities. Swimming, specifically exposure to heated swimming pools within the first year of life, significantly increased the rate of AIS development. It is believed that early chlorine neurotoxin exposure in the vaporization of chlorine in a swimming pool can exacerbate AIS development in younger children [36]. In 2014, Sperandio et al. [37] found that patients with AIS exhibited increased walking limitations and worse breathing patterns during exercise. However, researchers believe that increased walking-based aerobic activity will alleviate these symptoms, and these exercises should be encouraged.

\section{Double neuro-osseous theory disharmony}

Adolescent girls have higher rates of AIS development than their male counterparts. A four-part pathogenic speculative theory has been formulated: (1) the thoracospinal concept for right thoracic AIS in girls; (2) the new neuroskeletal biology relating the sympathetic nervous system to bone development; (3) white adipose tissue strengthening triglycerides and the role of leptin in energy homeostasis; and (4) leptin resistance in healthy females [38]. Thus, it is postulated that disharmony between somatic and autonomic nervous systems in the spine and trunk are exaggerated by hormonal dysregulation, resulting in skeletal overgrowth. Ultimately, it can result in physiological trunk width deviation or biomechanical disturbances in the skeletal framework.

First, the thoracospinal concept explains the asymmetrical growth in vascularity, breast size, skin temperature, and periapical rib length. It is believed that exacerbation of this asymmetry manifests as the biomechanical mechanism of pathogenesis, which is amplified during progressive growth.

Second, there has been evidence that hypothalamic leptin resistance occurs in juvenile females but not in males. It is believed to be an energy shunt to conserve energy for reproductive development. However, in some females, this process may be disrupted, resulting in increased central leptin sensitivity. In nonpathological individuals, two synchronous polarized processes called 
neuro-osseous timing of maturation escalators are balanced during normal growth and maturation: (1) osseous escalator and (2) neural escalator. The former relates to skeletal growth and shaping during development, whereas the latter explains the central nervous system adaptation and recalibration that occurs with growth to coordinate motor function. However, asynchrony or asymmetry during increased growth may result in pathology, due to disharmony in these two systems. The leptin sensitivity hypothesis is further supported by the negative correlation of BMI and AIS in adolescence.

Third, leptin level differences between males and females are significantly affected during puberty. With this dimorphism, females have $40 \%$ more leptin, which correlates with increased fat accumulation and adipose volume. Along with effects on adipose synthesis and metabolic effects, leptin plays a crucial role in bone formation and growth through the activation of the sympathetic system for skeletal growth. AIS has been strongly linked to increased leptin levels, which can be understood as dysregulation of symmetrical spinal growth, further explaining the increased AIS rate in females.

Thus, leptin resistance, resulting in leptin hypersensitivity, can incorrectly activate growth signals in the body resulting in disruptions of the osseous and neural escalators, which can exacerbate nonpathological asymmetries in females.

\section{Osteopenia}

AIS primarily manifests pathophysiological detriments preceding pubescent rapid growth in the early adolescent years. However, the symptoms can be seen before the manifestations of the classical symptoms. Bone mineral density (BMD) has been proposed as a prognostic factor of curve progression in patients with AIS. Runx2, a crucial gene in the regulation of osteoblast development and differentiation, was found to be significantly decreased in patients with reduced BMD $[39,40]$. In the study by Sun et al. [41], the use of BMD levels was explored as an independent risk factor in the progression of the disease and the duration of bracing treatment. Premenarcheal girls with lower BMD values were seen to have greater curve magnitudes, a lower Risser grade, and a main thoracic pattern [41]. Treatment duration and prognosis of the curve progression can be predicted before the exacerbation of spinal dysregulation during adolescent growth.
Lower BMD must also be considered for other circulating factors regulating peripheral and axial growth. Receptor activator of nuclear factor-kB ligand (RANKL) and osteocalcin serum measurements were observed at higher concentrations in adolescents with AIS. RANKL-to-osteoprotegerin (OPG) serum ratios were seen at higher levels in these individuals as well. Mouse models with RANKLto-OPG disruptions treated with genetic therapy displayed a significant reduction of AIS progression [24,42]. Dipeptidyl peptidase-4, which inactivates glucagon-like peptide-1 (GLP-1) and inhibits insulin secretion and sensitivity, was observed at higher levels in girls with AIS. GLP-1 increases insulin release in the pancreas, enhancing insulin response with bone growth during adolescent development. This further explains the increased prevalence of AIS in individuals with lower BMI values $[43,44]$.

Genetic and hormone therapy can be considered a new methodology of regulating the triggers of asymmetrical trunk bone development in the primary stages of AIS progression.

\section{Paraxial muscles}

The bilateral paraxial muscles are important in the symmetrical growth and development of spinal curvature. In patients with AIS, skeletal muscle and fatty deposition imbalances have been observed in the convex and concave facets of the curvature, respectively. Larger muscle volume on the convex side observed at the apex spinal level is consequential hypertrophy, because of stretch-induced stress on satellite cells. Furthermore, the fatty deposition on the opposite, concave facet of the curve is understood as fatty infiltration of muscle atrophy [45].

Muscle physiological differences in calmodulin levels further exacerbate the manifestations of spinal deformation. Calmodulin is observed at significantly higher levels on the convex side with lower levels on the concave facets of the spinal curvature [46]. A secondary messenger of the melatonin pathway and essential to muscle contraction, calmodulin is believed to play a role in the regulation of spinal alignment. In animal models, tamoxifen inhibition of calmodulin suppresses the natural process of scoliosis, thus indicating a possible reversal of the natural development of scoliosis [4]. The bidirectional relationship of bone and muscle pathophysiology is observed through this disruption of signal-regulated development and growth. 


\section{Conclusions}

AIS, while not fully understood, is rooted among genetic polymorphisms, physiological disruptions of signaling peptides and hormones, and environmental triggers. While not all these factors play a role in each patient, each patient with AIS can display some of these facets. To truly provide adequate treatment, the cause of the disease in each individual must be understood. Specifically, some treatments, such as bracing and tamoxifen, can be more useful in certain subsets of AIS than others. It must, therefore, be considered that AIS is not a cause of issues, but rather a syndrome revealing the disruptions of physiological homeostasis.

\section{Conflict of Interest}

No potential conflict of interest relevant to this article was reported.

\section{References}

1. Weinstein SL, Dolan LA, Wright JG, Dobbs MB. Effects of bracing in adolescents with idiopathic scoliosis. N Engl J Med 2013;369:1512-21

2. Andersen MR, Farooq M, Koefoed K, et al. Mutation of the planar cell polarity gene VANGL1 in adolescent idiopathic scoliosis. Spine 2017;42:E702-7.

3. Zhang Y, Gu Z, Qiu G. The association study of calmodulin 1 gene polymorphisms with susceptibility to adolescent idiopathic scoliosis. Biomed Res Int 2014;2014:168106.

4. Wu JZ, Wu WH, He LJ, et al. Effect of melatonin and calmodulin in an idiopathic scoliosis model. Biomed Res Int 2016;2016:8460291.

5. Zhao D, Qiu GX, Wang YP, Zhang JG, Shen JX, $\mathrm{Wu} \mathrm{ZH}$. Association between adolescent idiopathic scoliosis with double curve and polymorphisms of calmodulin1 gene/estrogen receptor- $\alpha$ gene. Orthop Surg 2009;1:222-30.

6. Buchan JG, Alvarado DM, Haller GE, et al. Rare variants in FBN1 and FBN2 are associated with severe adolescent idiopathic scoliosis. Hum Mol Genet 2014;23:5271-82.

7. Crijns TJ, Stadhouder A, Smit TH. Restrained differential growth: the initiating event of adolescent idiopathic scoliosis? Spine (Phila Pa 1976) 2017;42:E726-
32.

8. Haller G, Alvarado D, Mccall K, et al. A polygenic burden of rare variants across extracellular matrix genes among individuals with adolescent idiopathic scoliosis. Hum Mol Genet 2016;25:202-9.

9. Grauers A, Wang J, Einarsdottir E, et ak. Candidate gene analysis and exome sequencing confirm LBX1 as a susceptibility gene for idiopathic scoliosis. Spine J 2015;15:2239-46.

10. Zhu Z, Xu L, Leung-Sang Tang N, et al. Genomewide association study identifies novel susceptible loci and highlights Wnt/beta-catenin pathway in the development of adolescent idiopathic scoliosis. Hum Mol Genet 2017;26:1577-83.

11. Guo L, Yamashita H, Kou I, et al. Functional investigation of a non-coding variant associated with adolescent idiopathic scoliosis in zebrafish: elevated expression of the ladybird homeobox gene causes body axis deformation. PLoS Genet 2016;12:e1005802.

12. Xu JF, Yang GH, Pan XH, et al. Association of GPR126 gene polymorphism with adolescent idiopathic scoliosis in Chinese populations. Genomics 2015;105:101-7.

13. Li W, Li Y, Zhang L, et al. AKAP2 identified as a novel gene mutated in a Chinese family with adolescent idiopathic scoliosis. J Med Genet 2016;53:488-93.

14. Xu L, Huang S, Qin X, et al. Investigation of the 53 markers in a DNA-based prognostic test revealing new predisposition genes for adolescent idiopathic scoliosis. Spine 201515;40:1086-91.

15. Ryzhkov II, Borzilov EE, Churnosov MI, Ataman AV, Dedkov AA, Polonikov AV. Transforming growth factor beta 1 is a novel susceptibility gene for adolescent idiopathic scoliosis. Spine 2013;38:E699-704.

16. Xu L, Sun W, Qin X, Qiu Y, Zhu Z. The TGFB1 gene is associated with curve severity but not with the development of adolescent idiopathic scoliosis: a replication study in the Chinese population. BMC Musculoskelet Disord 2016;17:15.

17. Wang W, Ma J, Li SY, et al. Advance on genetic mechanism of adolescent idiopathic scoliosis and genetic relationship map. Zhongguo Gu Shang 2015;28:85460.

18. Roye BD, Wright ML, Williams BA, et al. Does ScoliScore provide more information than traditional clinical estimates of curve progression? Spine 2012;37:2099-103. 
19. Roye BD, Wright ML, Matsumoto H, et al. An independent evaluation of the validity of a DNA-based prognostic test for adolescent idiopathic scoliosis. J Bone Joint Surg Am 2015;97:1994-8.

20. Bohl DD, Telles CJ, Ruiz FK, Badrinath R, DeLuca PA, Grauer JN. A genetic test predicts providence brace success for adolescent idiopathic scoliosis when failure is defined a progression to $>45$ degrees. Clin Spine Surg 2016;29:E146-50.

21. Tang QL, Julien C, Eveleigh R, et al. A replication study for association of 53 single nucleotide polymorphisms in ScoliScore test with adolescent idiopathic scoliosis in French-Canadian population. Spine 2015;40:537-43.

22. Ogura Y, Kou I; Japan Scoliosis Clinical Research Group, et al. Genome-wide association study for adolescent idiopathic scoliosis. Clin Calcium 2016;26:553-60.

23. Simony A, Carreon LY, Hjmark K, Kyvik KO, Andersen MO. Concordance rates of adolescent idiopathic scoliosis in a Danish twin population. Spine 2016;41:1503-7.

24. Kulis A, Gozdzialska A, Drag J, et al. Participation of sex hormones in multifactorial pathogenesis of adolescent idiopathic scoliosis. Int Orthop 2015;39:122736.

25. Zhou C, Wang H, Zou Y, Fang H. Research progress of role of estrogen and estrogen receptor on onset and progression of adolescent idiopathic scoliosis. Zhongguo Xiu Fu Chong Jian Wai Ke Za Zhi 2015;29:1441-5.

26. Goultidis TT, Papavasiliou KA, Petropoulos AS, Philippopoulos A, Kapetanos GA. Higher levels of melatonin in early stages of adolescent idiopathic scoliosis: toward a new scenario. J Pediatr Orthop 2014;34:768-73.

27. Zamecnik J, Krskova L, Hacek J, Stetkarova I, Krbec M. Etiopathogenesis of adolescent idiopathic scoliosis: expression of melatonin receptors $1 \mathrm{~A} / 1 \mathrm{~B}$, calmodulin and estrogen receptor 2 in deep paravertebral muscles revisited. Mol Med Rep 2016;14:571924.

28. Yang P, Liu H, Lin J, Yang $H$. The association of rs4753426 polymorphism in the melatonin receptor 1B (MTNR1B) gene and susceptibility to adolescent idiopathic scoliosis: a systematic review and metaanalysis. Pain Physician 2015;18:419-31.
29. Yang M, Wei X, Yang W, et al. The polymorphisms of melatonin receptor 1B gene (MTNR1B) (rs4753426 and rs10830963) and susceptibility to adolescent idiopathic scoliosis: a meta-analysis. J Orthop Sci 2015;20:593-600.

30. Chen C, Xu C, Zhou T, et al. Abnormal osteogenic and chondrogenic differentiation of human mesenchymal stem cells from patients with adolescent idiopathic scoliosis in response to melatonin. Mol Med Rep 2016;14:1201-9.

31. Man GC, Wong JH, Wang WW, et al. Abnormal melatonin receptor $1 \mathrm{~B}$ expression in osteoblasts from girls with adolescent idiopathic scoliosis. J Pineal Res 2011;50:395-402.

32. Man GC, Wang WW, Yim AP, et al. A review of pinealectomy-induced melatonin-deficient animal models for the study of etiopathogenesis of adolescent idiopathic scoliosis. Int J Mol Sci 2014;15:1648499.

33. Burwell RG. Aetiology of idiopathic scoliosis: current concepts. Pediatr Rehabil 2003;6:137-70.

34. Burwell RG, Dangerfield PH, Moulton A, Grivas TB. Adolescent idiopathic scoliosis (AIS), environment, exposome and epigenetics: a molecular perspective of postnatal normal spinal growth and the etiopathogenesis of AIS with consideration of a network approach and possible implications for medical therapy. Scoliosis 2011;6:26.

35. Ramirez M, Martinez-Llorens J, Sanchez JF, et al. Body composition in adolescent idiopathic scoliosis. Eur Spine J 2013;22:324-9.

36. McMaster ME, Lee AJ, Burwell RG. Indoor heated swimming pools: the vulnerability of some infants to develop spinal asymmetries years later. Stud Health Technol Inform 2006;123:151-5.

37. Sperandio EF, Alexandre AS, Yi LC, et al. Functional aerobic exercise capacity limitation in adolescent idiopathic scoliosis. Spine J 2014;14:2366-72.

38. Burwell RG, Aujla RK, Grevitt MP, et al. Pathogenesis of adolescent idiopathic scoliosis in girls: a double neuro-osseous theory involving disharmony between two nervous systems, somatic and autonomic expressed in the spine and trunk: possible dependency on sympathetic nervous system and hormones with implications for medical therapy. Scoliosis 2009;4:24.

39. Wang WJ, Sun C, Liu Z, et al. Transcription factor Runx2 in the low bone mineral density of girls 
with adolescent idiopathic scoliosis. Orthop Surg 2014;6:8-14.

40. Wang S, Qiu Y, Ma Z, Xia C, Zhu F, Zhu Z. Expression of Runx 2 and type $X$ collagen in vertebral growth plate of patients with adolescent idiopathic scoliosis. Connect Tissue Res 2010;51:188-96.

41. Sun X, Wu T, Liu Z, et al. Osteopenia predicts curve progression of adolescent idiopathic scoliosis in girls treated with brace treatment. J Pediatr Orthop 2013;33:366-71.

42. Chiru M. Adolescent idiopathic scoliosis and osteopenia. Maedica (Buchar) 2011;6:17-22.

43. Normand E, Franco A, Moreau A, Marcil V. Dipeptidyl peptidase-4 and adolescent idiopathic scoliosis: expression in osteoblasts. Sci Rep 2017;7:3173.
44. Luciano RP, Wajchenberg M, Almeida SS, et al. Genetic ACE I/D and ACTN3 R577X polymorphisms and adolescent idiopathic scoliosis. Genet Mol Res 2016;15.

45. Jiang J, Meng Y, Jin X, et al. Volumetric and fatty infiltration imbalance of deep paravertebral muscles in adolescent idiopathic scoliosis. Med Sci Monit 2017;23:2089-95.

46. Acaroglu E, Bobe R, Enouf J, Marcucio R, Moldovan $\mathrm{F}$, Moreau A. The metabolic basis of adolescent idiopathic scoliosis: 2011 report of the "metabolic" workgroup of the Fondation Yves Cotrel. Eur Spine J 2012;21:1033-42. 